\title{
NONLINEAR ERROR CORRECT OF INTELLIGENT SENSOR BY USING GENETIC ALGORITHMS AND CUBIC SPLINE INTERPOLATION
}

\author{
Lin Lei, Houjun Wang and Yang Bai \\ School of Automation Engineering, University of Electronic Science and Technology of \\ China, Chengdu,610054 China
}

\begin{abstract}
The new technique in this paper is investigated to correct the nonlinear measurement error of intelligent sensor by use of genetic algorithms and cubic spline interpolation. The principle is using the advantage of both genetic algorithms and cubic spline function to reduce the iteration times and improve measurement precision of intelligent sensor. The way is through selecting genetic algorithms with appropriate design parameter and cubic spline function to build error correction model. The coefficients of the cubic spline function can be figured out by matrix operation. Then use genetic algorithms to fit the first part data, yielding optimization function and optimization coefficients. Simulation result and practice application show this technique is available compared with traditional way such as least-squares method.
\end{abstract}

Key words: GA, nonlinear error correction, cubic spline, optimization

\section{INTRODUCTION}

In system of industry control or automatic detection, the majority signals of electric voltage or electric current of sensors are the nonlinear. To nonlinear signal usually has two kinds of handling ways, one kind of ways is preparing the data form in advance according to the curve characteristic, then the adoption checks the form method to proceeds to handle to the signal of sensor; another method is nonlinear curve be divided into some a value for, each segment is linear or looking like for linear, according to the signal 
adopting kind getting, then find out the homologous linear zone, then makes use of the formula method handle to the signal. These two kinds of methods are all to orders the curvilinear cent segment to do in procedure, certainly data form can't be changed again. Obviously, above two kinds of methods contain a premise, that is this type of sensor signal of the curve a result for can having first, or saying getting of the same kind sensor must have good consistency, very much will have the error margin, even that gauge can't apply because the error margin is very big.

Aim at the problem as above, in this text we puts forward using genetic arithmetic combinative cubic spline interpolation method. Genetic Algorithms (GA) is especially suitable in solving complex and nonlinear problems, which are difficult for traditional searching algorithms. In practice intelligent sensor, nonlinear error of intelligent sensor is the source of most system. For the shortcoming of the nonlinear problem solving way existed presently, we present a new way combined genetic algorithms and cubic spline to correct nonlinear error. Spline interpolation function is in common use and important among those convergent and steady interpolation function. The cubic spline function with 2-order continue derivative is used under the condition of satisfying project design demands usually. It can be proved that the resolution of interpolation problems $\mathrm{S}(\mathrm{x})$ exists uniquely under the condition of above three boundary conditions.

\section{NONLINEAR ERROR CORRECTION MODEL}

In general, $\mathrm{x}$ expresses the physics parameters to be measured and $\mathrm{y}$ expresses the value measured by sensor. For $n$ groups experiment data $\left(x_{i}, y_{i}\right)$ $(\mathrm{i}=1,2, \ldots, \mathrm{n})$, we can adopt $\mathrm{m}$ term polynomial as the approximate function to describe these data: $y^{\prime}=a_{0}+a_{1} x+a_{2} x^{2}+\cdots+a_{m} x^{m}=\sum_{j=0}^{m} a_{j} x^{j}$
\[ A=\left(a_{0}, a_{1}, a_{2}, \cdots, a_{m}\right)^{T} \]

Where, $A=\left(a_{0}, a_{1}, a_{2}, \cdots, a_{m}\right)^{T}$ are $m+1$ coefficients to be confirmed.

Let $\delta_{i}$ be the error between the result figured out by formula (1) and $y_{i}$ at $x_{i}$. And import $n$ pairs measure data $\left(x_{i}, y_{i}\right)$ into formula (1), yielding $n$ equations: $\delta_{i}=y_{i}-y_{i}^{\prime}=y_{i}-\sum_{j=0}^{m} a_{j} x^{j}$

Where $\mathrm{i}=1,2 \ldots \mathrm{n}$. The goal is to get the optimum estimate value $A^{\prime}=\left(a_{0}^{\prime}, a_{1}^{\prime}, \cdots, a_{m}^{\prime}\right)^{T}$ under the condition of the least-squares sum of $\delta_{\prime}$. 
The individual fitness mapped from object function should be nonnegative. And the optimization direction should be the fitness increases direction. We use $E R$, the least-squares sum of $\delta_{i}$, as the object function in the process of dealing with nonlinear error.

$$
E R=\sum_{j=1}\left[\sum a_{j} x^{j}-y_{j}\right]^{2}
$$

The mapping relation between $f_{i}$ and object function $E R$ is:

$$
\begin{aligned}
& f_{i}(x)= \begin{cases}C_{\max }-E R, & E R<C_{\max } \\
0, & \text { others }\end{cases} \\
& C_{\max } \text { is an integer, which is maximum estimate value of } f(x) .
\end{aligned}
$$

Then use model as above to correct nonlinear error of sensor on-line. If the result of comparing with the value decided by model and real-time measurement by sensor, two of deviation beyond the reach of the normal motion scope that rule, precede again line revise. And this process can finish automatically by microprocessor in sensor.

\section{THE COMBINATION OF GENETIC ALGORITHMS AND CUBIC SPLINE}

Taking advantage of the GA and cubic spline function, we can reduce the iteration times and decrease the nonlinear error to zero at the boundary especially. The curve is very smooth at the joint of GA and cubic spline, and the correction precision will reach to a high level.

When we acquire a lot of measurement data by intelligent sensor in real measurement system. These data are divided into three parts unequally. Two points of intersection $\left(X_{1}, Y_{1}\right)$ and $\left(X_{2}, Y_{2}\right)$ will occur. We use GA to fit the first part data, yielding optimization function: $Y_{0}=A_{0}+B 0 X+C_{0} X_{2}+D_{0} X_{3}$

$A_{0}, \quad B_{0}, C_{0}$ and $D_{0}$ will be confirmed through many times of iteration of improved GA. The square sum of nonlinear error will be minimum. The second part data are fitted by cubic spline function. The coefficients of the cubic spline function can be figured out by matrix operation. This segment cubic spline function is: $\mathrm{Y}_{1}=\mathrm{A}_{1}+\mathrm{B}_{1} \mathrm{X}+\mathrm{C}_{1} \mathrm{X}_{2}+\mathrm{D}_{1} \mathrm{X}_{3}$

$$
\left[\begin{array}{cccc}
1 & x_{1} & x_{1}^{2} & x_{1}^{3} \\
0 & 1 & 2 x_{1} & 3 x_{1} \\
0 & 0 & 2 & 6 x_{1} \\
1 & x_{2} & x_{2}^{2} & x_{2}^{3}
\end{array}\right]\left[\begin{array}{c}
A_{1} \\
B_{1} \\
C_{1} \\
D_{1}
\end{array}\right]=\left[\begin{array}{c}
A_{0}+B_{0} x_{1}+C_{0} x_{1}^{2}+D_{0} x_{1}^{3} \\
B_{0}+2 C_{0} x_{1}+3 D_{0} x_{1}^{2} \\
2 C_{0}+6 D_{0} x_{1} \\
y_{2}
\end{array}\right]
$$


we get the third cubic spline function: $Y_{2}=A_{2}+B_{2} X+C_{2} X_{2}+D_{2} X_{3}$. According to the end point $\left(\mathrm{X}_{3}, \mathrm{Y}_{3}\right)$ of data , the matrix as follows:

$$
\left[\begin{array}{cccc}
1 & x_{2} & x_{2}^{2} & x_{2}^{3} \\
0 & 1 & 2 x_{2} & 3 x_{2}^{2} \\
0 & 0 & 2 & 6 x_{2} \\
1 & x_{3} & x_{3}^{2} & x_{3}^{3}
\end{array}\right]\left[\begin{array}{c}
A_{2} \\
B_{2} \\
C_{2} \\
D_{2}
\end{array}\right]=\left[\begin{array}{c}
A_{1}+B_{1} x_{2}+C_{1} x_{2}^{2}+D_{1} x_{2}^{3} \\
B_{1}+2 C_{1} x_{2}+3 D_{1} x_{2}^{2} \\
2 C_{1}+6 D_{1} x_{2} \\
y_{3}
\end{array}\right]
$$

The value of $A_{2}, B_{2}, C_{2}$ and $D_{2}$ can be figured out through matrix operation. Thus, three fitting curves have been figured out, and connected smoothly in turn. At the same time, the square sum of nonlinear error is minimum, satisfied the precision demand.

\section{GENETIC ALGORITHMS DESIGN}

The parameter of genetic algorithms has bigger influence on our correction algorithm, so we must design these parameters very well.

We adopt real number to encode the chromosome. Real number encoding mode can save the operation time, which has higher precision and explicit physics meaning. adopt 2 member sign gene table $S=\{+,-\}$ and 11 member numerical value gene table $\mathrm{V}=\{0,1,2,3,4,5,6,7,8$, 9 , - to encode chromosome, where ". " expresses radix point. Letters with subscript can express every chromosome, where subscript expresses sequence of location. The sign gene takes part in the mutation operation. The sign "+" can mutates only to "-" , and the sign " -" can mutates only to "+" . Adopting this encoding rule, invalidation pattern will not occur in chromosome individuals no matter what selection, crossover or mutation operation.

The fitness distribute strategy adopted in this paper is based on sorting. The population is sorted according to the object value. Sorting method imported with population uniformity scale supplies a simple and effective way to control the selection pressure. The robustness of sorting method is better than that of proportion method.

We give attention to global and local searching ability through selecting high crossover probability and mutation probability synthetically. Such pair of operation has both cooperation and competition. The design and realization of crossover operator relates to special problem. We adopt simple single point crossover operator at here. The defining domain is determined by vector $A=\left(a_{1}, a_{2}, \cdots, a_{i}, \cdots a_{n}\right) T$ and $B=\left(b_{1}, b_{2}, \cdots, b_{i}, \cdots b_{n}\right) T$. In order to make sure $D_{n}=A \times B$, namely the solution domain is protruding domain, we make any chromosome $X 0$ satisfy $\forall i \in\{1,2, \cdots n\}, a_{i} \leq x_{i 0} \leq b_{i}$. Let the two chromosomes to be crossed in parent generation are: $X=\left(x_{1}, x_{2}, \cdots, x_{i} \cdots x_{n}\right)^{T}$, 
$Y=\left(y_{1}, y_{2}, \cdots, y_{i} \cdots y_{n}\right)^{T}$.For any $i$, if $x_{i} \leq y_{i}$ (or else, exchange $x_{i}$ and $\left.y_{i}\right)$, thus the cross algorithms defined as follows: $\mathrm{C}=\mathrm{A} \oplus \mathrm{B}$

$$
\mathrm{C}_{\mathrm{i}}=\left\{\begin{array}{l}
\alpha a_{i}+(1-\alpha) x_{i} \bmod (\theta, 4)=0 \\
\alpha x_{i}+(1-\varepsilon) y_{i} \bmod (\theta, 4)=1 \text { or } 2 \quad(\mathrm{i}=1,2, \cdots, \mathrm{n}) \\
\alpha y_{i}+(1-\alpha) b_{i} \bmod (\theta, 4)=3
\end{array}\right.
$$

where, $\alpha$ is a random real number satisfied $\alpha \in[0,1]$ and $\theta$ is a random nonnegative integer. In above formula, the probability, which the value of child is between parents, equals to that of other two instances, so that better convergence speed can be achieved when the solutions are inner points.

According to the characteristics of protruding combination, if $X, Y \in D_{11}$, $C \in D_{n}$, what can make sure the searching proceed in certain space. Therefore, the cross operation of evolution GA can assure the solutions to be better and better and make the evolution GA has more strong global convergence ability that can deal with not only inner point problem but also the problem with solutions located near the boundary.

Improved mutation operation is: $\mathrm{C}=\nabla_{\theta} \mathrm{X}$

$\mathrm{C}_{\mathrm{i}}=\left\{\begin{array}{l}\alpha a_{i}+(1-\alpha) x_{i} \bmod (\theta, 2)=0 \\ \alpha x_{i}+(1-\alpha) b_{i} \bmod (\theta, 2)=1\end{array} \mathrm{X} \in \mathrm{D}_{\mathrm{n}}, \quad \forall \mathrm{x}_{\mathrm{i}} \in \mathrm{X}\right.$

$\alpha$ is a random real number; satisfied with $0 \leq \alpha \leq 1$, and $\theta$ is a random non-negative integer.

\section{SIMULATION RESULTS}

The example of nonlinear error correction is based on thermocouple temperature intelligent sensor in this paper. The GA approach equation of the first segment is: $F(X)=3.4977+2.5038 X+1.4984 \mathrm{X}_{2}+0.5002 \mathrm{X}_{3}$

The sum of error square is $3.2804 \mathrm{e}-006$, and the maximum relative error is 9.9781e-005. Simulation software is programmed by MATLAB language. We can get the coefficients of the approximation equation of the second segment through importing the coefficients of the first segment into this program. The coefficients of the third segment, the fourth segment, $\cdots$, the sixteenth segment may be deduced by analogy. Here, the square sum of error of total group data is 5.4039e-006, and the curve of simulation shows in Fig1.In Fig1, horizontal coordinate is temperature $\left({ }^{\circ} \mathrm{C}\right)$, vertical coordinate is voltage (unit is $\left.\mu \mathrm{V}\right)$.

In addition, the result of this method is better than least squares method and the best uniform approximation compared with traditional ways, and the precision is better than that of genetic algorithms purely. 


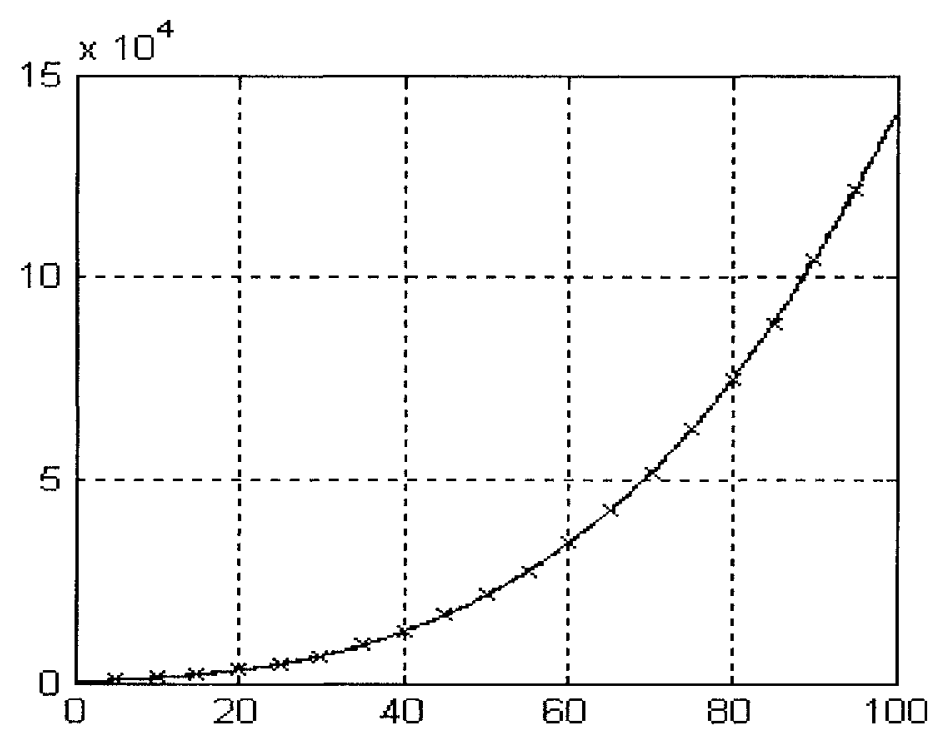

Figure 1. Facilities

\section{CONCLUSIONS}

We researched the ability of combination GA and cubic spline interpolation to correct nonlinear error of intelligent sensor. Data information that makes use of the hour of normal movement in sensor can reflect the normal measurement data of sensor. Like this correction curve or importations that establishes output the relation since solved the inconsistent of the sensor, increases measurement accuracy again. The simulation and practice application show the feasibility of combination genetic algorithms and cubic spline interpolation to correct nonlinear errors of sensor, put forward to the sensor measurement data in the text not the line error margin revises the technique, for all kinds of signals are all containing good result, having the application the value.

\section{REFERENCES}

[1]Li Qing-Yang, Guan Zhi, Bai Feng-Shan, numerical value compute theory, Beijing: Tsinghua university Publishing House, 2000.

[2]Wang Xiao-Ping, Cao Li-Ming, genetic algorithms - theory, application and software realization, Xi'an: Xi' an Jiaotong university Publishing House, 2002.

[3]Zhou Ming, Sun Shudong, genetic algorithms theory and application, Beijing: national defense industry Publishing House, 1999. 